Abstract PTU-18 Table 1 Endoscopy workfore by procedure

\begin{tabular}{|c|c|c|c|c|c|c|}
\hline & Role & Endoscopists & Procedures & $\begin{array}{l}\text { Median Endoscopist } \\
\text { Annual Procedures } \\
\text { (IQR) }\end{array}$ & $\begin{array}{l}\text { Endoscopists } \\
\text { performing fewer } \\
\text { than recommended } \\
\text { procedures annually* }\end{array}$ & $\begin{array}{l}\text { Procedures by Endoscopists } \\
\text { performing fewer than } \\
\text { recommended annually (\% } \\
\text { total procedures) }\end{array}$ \\
\hline \multirow[t]{3}{*}{ OGD } & Doctor & 2937 (89.5\%) & 404529 (76.9\%) & $94(23-218)$ & $51.3 \%$ & \\
\hline & $\mathrm{NE}$ & 345 (10.5\%) & $121249(23.1 \%)$ & $243(23-548)$ & $36.2 \%$ & \\
\hline & Total & 3282 & 525778 & $101(23-240)$ & $49.7 \%$ & $48566(9.2 \%)$ \\
\hline \multirow[t]{3}{*}{ Colon } & Doctor & 2567 (89\%) & $357853(83.8 \%)$ & $110(30-221.5)$ & $47.1 \%$ & \\
\hline & NE & $317(11 \%)$ & $69327(16.2 \%)$ & $170(16-343)$ & $40.1 \%$ & \\
\hline & Total & 2884 & 427180 & $113.5(28-232.25)$ & $46.3 \%$ & $42842(10 \%)$ \\
\hline \multirow[t]{3}{*}{ FS } & Doctor & $2516(87.3 \%)$ & $141909(55.2 \%)$ & $37(12-76)$ & na & \\
\hline & $\mathrm{NE}$ & $365(12.7 \%)$ & $115279(44.8 \%)$ & $173(46-497)$ & na & \\
\hline & Total & 2881 & 257188 & $41(13-90)$ & na & na \\
\hline ERCP** & Total & 316 & 20676 & $63.5(13-104)$ & $55.1 \%$ & $4308(20.8 \%)$ \\
\hline
\end{tabular}

ERCPs, but these endoscopists perform only 9.8\% of total procedures. Further analysis is required to compare KPIs for those not reaching and those exceeding the minimums. If other KPIs are low in the group not reaching the minimum additional intervention is required; conversely, if this is not the case, the appropriateness of the minimum standard should be reviewed.

\section{PTU-19 THE ENDOSCOPY SAFETY ATTITUDES QUESTIONNAIRE (ENDO-SAQ): RESULTS OF A PILOT STUDY}

1,2,3 Srivathsan Ravindran* ${ }^{1}$ Chris Healey, 1,2Sarah Marshall, ${ }^{1}$ Mark Coleman ${ }^{3}$ Hutan Ashrafian, ${ }^{3}$ Ara Darzi, ${ }^{2,3}$ Siwan Thomas-Gibson. 'Joint Advisory Group On Gi Endoscopy (jag), London, UK; ' Wolfson Unit for Endoscopy, St Mark's Hospital, London, UK; ${ }^{3}$ Imperial College London, London, UK

\subsection{6/gutjnl-2021-BSG.92}

Introduction Across healthcare, evidence has demonstrated the influence of safety culture on patient outcomes. We developed a novel tool to measure endoscopy safety culture - the Endoscopy Safety Attitudes Questionnaire (Endo-SAQ). Our aims were to assess the safety attitudes of our workforce and tool validity in a pilot study.

Methods The previously validated SAQ was adapted by the core research team to reflect endoscopy-specific content. An expert group was convened to undertake a rating exercise to assess content validity. Endo-SAQ has 6 domains: teamworking, safety climate, job satisfaction, stress recognition, perceptions of management and working conditions.

The Endo-SAQ was administered to the staff at a tertiary UK endoscopy unit. Outcome measures were mean domain scores, percentage of positive responses per domain, content validity indices (item (I-CVI) and scale (S-CVI)) and internal consistency (Cronbach's alpha). Statistical analysis included descriptive statistics, paired samples $t$ test, one-way ANOVA and Kruskal-Wallis tests.

Results 61 participants completed the survey (completion rate $88.4 \%$ ). All domains scores were over $60 \%$, indicating a positive overall response across domains. The percentage of positive responses per domain varied, with 'perceptions of management' and 'working conditions' both scoring $<40 \%$ (Table 1). This appears to be in line with the wider literature
Abstract PTU-19 Table 1 Mean scores (as\%) and percentage of positive responses for each domain (SD = standard deviation)

\begin{tabular}{lll}
\hline Domain & Mean score (SD) & \% positive responses \\
\hline Teamworking & $74.32(20.75)$ & 59.70 \\
Safety climate & $76.13(16.16)$ & 58.70 \\
Job satisfaction & $77.68(19.07)$ & 66.70 \\
Stress recognition & $73.51(26.89)$ & 66.70 \\
Perceptions of management & $64.35(22.44)$ & 33.90 \\
Working conditions & $66.78(19.77)$ & 38.70 \\
\hline
\end{tabular}

in medicine and surgery. On subgroup analysis, nurses scored 'job satisfaction' $(p=0.01)$, 'perceptions of management' ( $p$ $=0.002)$ and 'working conditions' $(p=0.004)$ significantly lower than endoscopists.

Five independent expert raters scored the Endo-SAQ for clarity and relevance. Four items had an I-CVI $<0.78$ resulting in question rewording. Overall, S-CVI was $>0.90$ indicating good content validity. All Endo-SAQ domains scored 'acceptable' or above for internal consistency.

Conclusions Endo-SAQ can detect safety attitudes and differences between groups. A national study is due to be undertaken utilising Endo-SAQ in addressing modifiable factors to support the workforce better.

\section{PTU-20 NO SURVEILLANCE INTERVAL CHANGE WITH OPTICAL DIAGNOSIS OF SMALL POLYPS DURING BOWEL CANCER SCREENING COLONOSCOPY}

Ahmir Ahmad*, Ana Wilson, Angad Dhillon, Siwan Thomas-Gibson, Noriko Suzuki, Adam Humphries, Adam Haycock, Kevin Monahan, Margaret Vance, Brian Saunders. Wolfson Unit for Endoscopy, Imperial College London, London, UK

\subsection{6/gutjnl-2021-BSG.93}

Introduction High confidence optical diagnosis (OD) in combination with a 'resect and discard strategy' during screening colonoscopy has advantages over histology alone, provided accuracy is maintained regarding surveillance intervals. Low confidence diagnoses, and/or polyps $>1 \mathrm{~cm}$ still require histology to complete individual patient polyp datasets and inform 